Article

\title{
SARS-CoV-2 mRNA Vaccine Immunogenicity in Hemodialysis Patients: Promising Vaccine Protection That May Be Hindered by Fluid Overload
}

\author{
Hedia Hebibi 1,2,*(D), Marvin Edeas ${ }^{3} \mathbb{D}$, Laure Cornillac ${ }^{4}$, Severine Beaudreuil ${ }^{2}$, Jedjiga Achiche ${ }^{1}$, David Attaf ${ }^{4}$, \\ Samah Saibi ${ }^{1}$, Charles Chazot ${ }^{4}$, Fatah Ouaaz ${ }^{5,+}$ and Bernard Canaud ${ }^{6,+}$ \\ 1 NephroCare Ile de France, 94800 Villejuif, France; Jedjiga.achiche@fmc-ag.com (J.A.); \\ Samah.saibi@fmc-ag.com (S.S.) \\ 2 Néphrologie Dialyse et Transplantation CHU Bicêtre, 94275 Le Kremlin Bicêtre, France; \\ severine.beaudreuil@aphp.fr \\ 3 Department Endocrinology, Metabolism and Diabetes, Institut Cochin, INSERM U1016-CNRS UMR \\ 8104-Université de Paris, 75014 Paris, France; marvin.edeas@inserm.fr \\ 4 Fresenius Medical Care France, 94260 Fresnes, France; laure.cornillac@fmc-ag.com (L.C.); \\ David.attaf@fmc-ag.com (D.A.); Charles.chazot@fmc-ag.com (C.C.) \\ 5 Institut Cochin, INSERM, CNRS, Université de Paris, 75014 Paris, France; fatah.ouaaz@inserm.fr \\ 6 France and Fresenius Medical Care Deutschland, Global Medical Office, Montpellier University, Montpellier, \\ DE-61352 Bad Homburg, Germany; Bernard.canaud@fmc-ag.com \\ * Correspondence: hebibiel-hadia@fmc-ag.com; Tel.: +33-146788081; Fax: +33-146770269 \\ + These authors contributed equally to this work.
}

Citation: Hebibi, H.; Edeas, M.; Cornillac, L.; Beaudreuil, S.; Achiche, J.; Attaf, D.; Saibi, S.; Chazot, C.; Ouaaz, F.; Canaud, B. SARS-CoV-2 mRNA Vaccine Immunogenicity in Hemodialysis Patients: Promising Vaccine Protection That May Be Hindered by Fluid Overload. Kidney Dial. 2022, 2, 44-56. https://doi.org/ 10.3390/kidneydial2010006

Academic Editors: Massimo Torreggiani and Giuliano Brunori

Received: 18 December 2021

Accepted: 18 January 2022

Published: 28 January 2022

Publisher's Note: MDPI stays neutral with regard to jurisdictional claims in published maps and institutional affiliations.

Copyright: (C) 2022 by the authors. Licensee MDPI, Basel, Switzerland. This article is an open access article distributed under the terms and conditions of the Creative Commons Attribution (CC BY) license (https:// creativecommons.org/licenses/by/ $4.0 /)$.

\begin{abstract}
Background: Due to their immunocompromised conditions, hemodialysis (HD) patients are at high risk of being infected with SARS-CoV-2 with poor clinical outcomes. We explored safety, efficacy and variability factors associated with vaccine immune response in these patients. Methods: From 18 January to 30 April 2021, 87 HD patients were enrolled in this study and subdivided in two sub-groups: SARS-CoV-2 positive prior to vaccination and naïve patients. The vaccination protocol included two intramuscular shots of the mRNA vaccine at a 28-day interval. The vaccine response was evaluated one month after the first and second shots by measuring anti-S antibody titers. Findings: $44 \%$ of the patients studied, were women; median age was 58 years. One month after the first shot, $64.3 \%$ of patients had anti-S antibody titers $<50 \mathrm{U} / \mathrm{mL}$ while $96.5 \%$ had anti-S antibody titers $>250 \mathrm{U} / \mathrm{mL}$ one month after the second shot. We show that the anti-SARS-CoV-2 spike mRNA vaccine elicits a great tolerability in HD patients. Importantlly, the vaccine response is characterized by a higher rate of seroconversion than conventional vaccination, as we have shown for the hepatitis B vaccine. We highlight two major factors of variability of the vaccine response. First, pre-exposure to SARS-CoV-2 boosts humoral response, as $95 \%$ of SARS-CoV-2 positive patients developed anti-S antibody titers > $250 \mathrm{U} / \mathrm{L}$ just after the first shot. Secondly, on the contrary fluid overload strikingly appears, limiting the vaccine response, as $84 \%$ of HD patients with fluid overload elicit lower anti-S antibody titers after the first shot. Interpretation: Our findings show that the immunogenic profile induced by mRNA vaccines in HD patients represents a promising a protective strategy that is associated with greater tolerability. Fluid overload may be considered as a novel factor that hinders the immune response in HD patients and further studies will be needed to investigate this working hypothesis.
\end{abstract}

Keywords: mRNA vaccine; COVID-19; antibody against SARS-CoV-2 spike protein; hemodialysis; hemodiafiltration; fluid overload; lymphocyte; adverse events; T cells; B cells

\section{Introduction}

In 2020, COVID-19 became the third leading cause of death worldwide, with a particularly devastating effect on chronic kidney disease (CKD) patients. Due to ambulatory and 
repeated life support treatment conditions, hemodialysis (HD) patients are more exposed to SARS-CoV-2 infection than the general population, as they cannot comply with lockdown measures due to repeated use of transportation to dialysis units, including mingling with other patients and caregivers. In addition, due to their comorbid conditions and immunodeficient profile, HD patients are particularly vulnerable [1] and have a higher risk of death from SARS-CoV-2 infection, which ranges from $16 \%$ to $32 \%$ [2-4]. The development of vaccines based on messenger RNA biotechnology offers an innovative approach for overcoming poor outcomes of COVID-19 infection and the related complications in HD patients [5]. Messenger RNA (mRNA) vaccines relying on an innovative technology that combines molecular biology and immunology have shown unexpectedly strong results $[5,6]$.

BNT162b2 (Comirnaty ${ }^{\mathrm{TM}}$, Pfizer/BioNtech) was one of the first vaccines approved worldwide for COVID-19 protection. A large study in a healthy population showed that a two-dose vaccination procedure reduced severe acute respiratory syndrome and subsequent death by greater than $90 \%$ [7]. However, due to their comorbidities dialysis patients were excluded from this pilot study [8], meaning that the immunogenic profile of mRNA vaccines in this population is poorly understood. In addition, it is well established that the immune response to vaccines is impaired in HD patients, who exhibit lower seroconversion rates than subjects with normal kidney function [9]. Interestingly, a large proportion of HD patients may develop asymptomatic COVID-19 disease, as indicated by the reported high prevalence of positive antibodies against SARS-CoV-2 in two large dialysis units [10]. A vaccine campaign was launched in France in January 2021, and dialysis patients were targeted as a priority population for receiving the SARS-CoV-2 vaccine. We took advantage of this decision by health authorities to evaluate the tolerance and immune response of patients in our dialysis facility.

All patients signed consent and the study approval was obtained internally. This study had three aims: first, to assess tolerance of HD patients to the vaccine; second, to evaluate the immune response by monitoring antibody titers against SARS-CoV-2 spike (Anti-S) protein after two shots of the Comirnaty ${ }^{\circledR}$ mRNA vaccine by Pfizer BioNtech; and third, to compare antibody titers (anti-S) in HD patients to healthy controls, while identifying factors associated with immune response.

\section{Materials and Methods}

\subsection{Patients and Healthy Controls}

Ninety-eight patients on maintenance in center hemodialysis and short daily home hemodialysis (SDHD) were enrolled in this prospective study from 18 January to 30 April 2021. All patients received appropriate information about the protocol and signed the consent form. Three patients who had symptomatic COVID-19 infection within the last 3 months were excluded from the study. A fourth additional patient bearing multiple sclerosis was contraindicated for the vaccine. Another five patients refused to be vaccinated. Our cohort was subdivided into two subgroups: symptomatic and asymptomatic COVID19 group (COVID-19 positive) and the naïve patient group (COVID-19 negative).

Twenty-three healthy volunteers consisting of naïve to COVID-19 caregivers who were vaccinated using the same two-shot vaccine protocol within the same time frame represented the control group.

\subsection{Assessment of Biological Parameters}

Monthly blood samples were drawn before the dialysis session to assess routine laboratory parameters (albumin, C-reactive protein, hemoglobin, platelets, and white blood cell count). Blood samples were drawn to assess the immune vaccination response after the first and second vaccine shot. Every 3 months, as routine practice, screening for hepatitis B (HBV antigen, Ac anti-HBs) status is performed in our dialysis facility. All HD patients had been vaccinated against hepatitis $B$, and anti-HBS antibody titers were regularly monitored. 


\subsection{Vaccine Protocol}

The vaccine protocol followed the recommendations of the health authority and the manufacturer's instructions. All the patients received two intramuscular injections of $30 \mu \mathrm{g}$ $(0.3 \mathrm{~mL})$ of the Comirnaty ${ }^{\circledR}$ BNT162 b2 mRNA Pfizer BioNtech vaccine in the deltoid muscle at 28-day intervals.

\subsection{Evaluation of the Antibody Response}

As part of our routine practice, systematic SARS-CoV-2 RNA screening is performed by RT-PCR from nasopharyngeal swabs using the Alinity m SARS-CoV-2 assay (Abbott Diagnostics, Chicago, IL, USA) when a SARS-CoV-2 infection is suspected. In addition, at baseline, and before the first vaccine shot, SARS-CoV-2 serological test was performed with IgG and IgM antibodies test (Abbott) (IgG negative $<50 \mathrm{IU} / \mathrm{mL}$, IgM positive $>1$ ). The Elecsys Anti-SARS-CoV-2, and the Elecsys Anti-SARS-CoV-2 S immunoassays (Roche Diagnostics, Rotkreuz, Switzerland) were performed one month after the first and second vaccine shots. This technique was used for the qualitative detection of antibodies to SARS-CoV-2 nucleocapsid protein $(\mathrm{N})$ and the quantitative determination of antibodies to the SARS-CoV-2 spike (S) protein receptor binding domain (RBD). Anti-N antibodies are considered detected when the assay index result is $>1$. Anti-S titers $<0.4 \mathrm{U} / \mathrm{mL}$ are considered non-reactive. The cut-off for seroconversion in the assay is $50 \mathrm{U} / \mathrm{mL}$. The threshold of $250 \mathrm{U} / \mathrm{mL}$ is a maximum level for detecting anti-S antibodies. We classified anti-S titers into 4 categories: $<0.4,0.4-50,50-250$, and $>250 \mathrm{U} / \mathrm{mL}$. The immune response was evaluated 28 days after the first and second shots.

\subsection{Data Collection}

Patient data were captured from our electronic medical record (EMR): demographic data and comorbidities, the Charlson comorbidity index, body mass index (BMI), and other notable past medication.

Blood laboratory tests at baseline and one month after the first and second shots were collected for patients and members of the control group. Dry weight and fluid status in HD patients were monitored by multifrequency bioimpedance (body composition monitor, BCM: Fresenius Medical Care) measurements before first vaccine shot and at 3-month intervals. The dialysis dose was assessed monthly by single-pool Kt/V (spKt/V) for thrice weekly treatment and standard weekly Kt/V (sdwkKt/V) for patients on short daily home hemodialysis (SDHH). Adverse effects of vaccines were defined as follows: site-injection pain, skin redness, fever $>38$, myalgia, arthralgia, headache, fatigue, and diarrhea. These events were recorded during the study period.

\subsection{Statistical Analysis}

All statistical analyses were performed using R Core Team (2020). R: A language and environment for statistical computing. R Foundation for Statistical Computing, Vienna, Austria (version 1.4.1103). The data were first analyzed using descriptive statistics. The average values, standard deviations and percentages of the different data features are summarized in the indicated tables and figures. Mean and standard deviation were computed for numerical data and counts and percentages for categorical features. Counts and percentages of grouped data are represented using boxplots and bar plots, as shown in figures. Inferential tests were performed to determine specific data features, and normality was tested using the Shapiro method and quantile-quantile plots. Since none of the tests confirmed that our data features were normally distributed, we compared the medians of observations using the Mann-Whitney-Wilcoxon test for independent samples and the Friedman test for dependent samples. The independence between different data variables was tested using the chi-squared test, and correlations between parameters were assessed by Spearman analysis. In all our analyses, a $p$-value of 0.05 was considered statistically significant. We calculated the $95 \%$ confidence intervals for the mean differences of the two 
cohorts using Student's $t$-test and Welch correction (whenever the variances were not equal; this last assumption was investigated using the Fisher test).

\section{Results}

\subsection{Patients and Control Group Characteristics}

Eighty-seven HD patients and twenty-three healthy volunteers, as a control group, were included in this analysis. The dialysis population was split into two groups, as illustrated in the study flow chart in Figure 1. Demographic and patient characteristics are summarized in Table 1.

\begin{tabular}{|c|c|c|}
\hline \multicolumn{3}{|c|}{ Study population } \\
\hline Control group $(n=23)$ & \multicolumn{2}{|c|}{ Patients group ( $n=98$ ) } \\
\hline$\nabla$ & 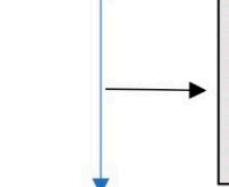 & $\begin{array}{l}n=5 \text { patients refused the vaccination } \\
n=4 \text { patients excluded from the study } \\
\text { ( } 3 \text { symptomatic COVID- } 19<3 \text { months, } \\
1 \text { vaccine contraindication) }\end{array}$ \\
\hline \multicolumn{3}{|c|}{$\begin{array}{c}\text { M0: BNT162b2 mRNA Vaccination } \\
1 \text { st shot } \\
\text { Baseline serology (IgM, IgG): }\end{array}$} \\
\hline $\begin{array}{c}\text { Control group }(\mathbf{n}=\mathbf{2 3}) \\
\text { (2 symptomatic COVID-19 }>3 \text { months, } \\
2 \text { asymptomatic, } 2 \text { Naïve) }\end{array}$ & \multicolumn{2}{|c|}{$\begin{array}{c}\text { Patients group ( } \mathbf{n}=\mathbf{8 9} \text { ) } \\
\text { (7 Symptomatic COVID-19 }>3 \text { months, } \\
12 \text { asymptomatic COVID-19, } 70 \text { Naive) }\end{array}$} \\
\hline & & $\begin{array}{l}\mathrm{n}=2 \text { patients excluded } \\
\quad(1 \text { death, } 1 \text { renal transplantation) }\end{array}$ \\
\hline
\end{tabular}

28 days post- 1 st shot

\section{M1: BNT162b2 mRNA Vaccination \\ 2 nd shot \\ Vaccine response assessment post-1 st shot}

\begin{tabular}{|c|c|}
\hline $\begin{array}{c}\text { Control group ( } \mathbf{n}=\mathbf{2 3} \text { ) } \\
\text { (2 symptomatic COVID-19 }>3 \text { months, } \\
2 \text { asymptomatic, 2 Naïve) }\end{array}$ & $\begin{array}{c}\text { Patients group ( } \mathbf{n = 8 7} \text { ) } \\
\text { (7 Symptomatic COVID-19 }>3 \text { months, } \\
12 \text { asymptomatic COVID-19, 68 Naive) }\end{array}$ \\
\hline M2: BNT162b2 mRNA Vaccine response assessment post-1 st $+2 \mathrm{nd}$ \\
shots
\end{tabular}

Figure 1. Study flow chart.

Thirty-eight patients were women (44\%), and the median age was 58 years $(58.36 \pm 14.93)$. The Charlson comorbidity index was $4.13 \pm 1.18$, with a body mass index (BMI) of $27.06 \pm 5.66 \mathrm{~kg} / \mathrm{m}^{2}$. Of all of the patients, $3 \%$ were on immunosuppressive therapy. The median age of the control group was 45 years with a body mass index (BMI) of $24 \mathrm{~kg} / \mathrm{m}^{2}$. Renal replacement therapy consisted of three weekly treatments of high-flux hemodialysis in $51 \%$, high-volume hemodiafiltration in $42 \%$ with a mean convective volume of $24.8 \mathrm{~L}$ $(24.80 \pm 3.05)$, while five patients $(6 \%)$ were on short daily home hemodialysis. The mean 
weekly treatment time was $743.45 \pm 39.93 \mathrm{~min}$. Sixty-eight $(78.2 \%)$ patients and nineteen healthy controls $(82.6 \%)$ had not contracted the COVID-19 infection beforehand.

Table 1. Baseline characteristics.

\begin{tabular}{|c|c|c|}
\hline Parameters & Dialysis Group & Control Group \\
\hline \multicolumn{3}{|l|}{ Demographic (median, 25th-75th percentile) } \\
\hline Age (year) & $60(49.5-69)$ & $45(35-55)$ \\
\hline Sex count (Male/Female) & $49 / 38$ & $9 / 15$ \\
\hline Dry Body Weight $(\mathrm{kg})$ & $74(61.8-83)$ & $66(56-71)$ \\
\hline Height $(\mathrm{cm})$ & $166(163-170.5)$ & $167(164-168)$ \\
\hline Body mass index (BMI: $\mathrm{kg} / \mathrm{m}^{2}$ ) & $25.4(22.7-31.6)$ & $24(23-26)$ \\
\hline \multicolumn{3}{|l|}{ COVID-19 History (n, \%) } \\
\hline Symptomatic & $7(8.0 \%)$ & $3(13.0 \%)$ \\
\hline Asymptomatic & $12(13.8 \%)$ & $1(4.3 \%)$ \\
\hline None & $68(78.2 \%)$ & $19(82.6 \%)$ \\
\hline \multicolumn{3}{|l|}{ Comorbidities (n, \%) } \\
\hline Charlson Index & $4.13 \pm 1.18$ & \\
\hline Diabetes & $16(18.39 \%)$ & $0(0.00 \%)$ \\
\hline Hypertension & $85(97.70 \%)$ & $2(8.69 \%)$ \\
\hline \multicolumn{3}{|l|}{ Medication (n, \%) } \\
\hline Antihypertensive & $80(91.95 \%)$ & - \\
\hline Cardiovascular medication & $38(43.68 \%)$ & - \\
\hline Phosphate binders & $58(66.67 \%)$ & - \\
\hline Carbonat of calcium & $50(57.47 \%)$ & - \\
\hline Cholecalciferol & $85(97.70 \%)$ & - \\
\hline Iron & $87(100.0 \%)$ & - \\
\hline Vitamin $\mathrm{K}$ antagonist & $5(5.75 \%)$ & - \\
\hline \multicolumn{3}{|l|}{ Nephropathy (n, \%) } \\
\hline Glomerular & $1(1.15 \%)$ & - \\
\hline Vascular & $62(71.26 \%)$ & - \\
\hline Diabetic & $15(17.24 \%)$ & - \\
\hline Autosomal Polycystic Kidney Disease & $3(3.45 \%)$ & - \\
\hline HIV & $3(3.45 \%)$ & - \\
\hline Other & $11(12.64 \%)$ & - \\
\hline \multicolumn{3}{|l|}{ Renal Replacement Therapy } \\
\hline Dialysis vintage (Months) & $45.19 \pm 37.39$ & - \\
\hline Past History of renal transplantation (n, \%) & $8(9.19 \%)$ & - \\
\hline Immunossupressive therapy past/active (n, \%) & $3(3.45 \%)$ & - \\
\hline
\end{tabular}

\subsection{Biological Parameters Assessment}

Laboratory test parameters at baseline and after the first and the second shots are shown in Tables 2 and 3 Before vaccination, the median hemoglobin value was $11 \mathrm{~g} / \mathrm{dL}$ (10.3-11.65), the median albumin level was $39 \mathrm{~g} / \mathrm{L}(39.88 \pm 3.33)$ and the median leukocyte count was $6000 / \mathrm{mm}^{3}(5048-7588)$. As shown in Table 5, ten patients had HBS antibody hepatitis virus titers $<10 \mathrm{IU} / \mathrm{L}$. 
Table 2. Hemodialysis Parameters.

\begin{tabular}{|c|c|}
\hline Hemodialysis Parameters & \\
\hline \multicolumn{2}{|l|}{ Treatment Schedule and Modality } \\
\hline Weekly treatment time (min/week) & $743.45 \pm 39.93$ \\
\hline High flux HD mode (\%) & $87(100 \%)$ \\
\hline Conventional High Flux HD (n, \%) & $45(51.72 \%)$ \\
\hline $\operatorname{HDF}(n, \%)$ & $37(42.53 \%)$ \\
\hline Short Daily Home HD (n, \%) & $5(5.75 \%)$ \\
\hline Anticoagulation (IU/session) & $2686.21 \pm 1272.97$ \\
\hline \multicolumn{2}{|l|}{ Vascular access } \\
\hline Native Arteriovenous Fistula (n, \%) & $81(93.10 \%)$ \\
\hline Central Venous Catheter (n, \%) & $6(6.90 \%)$ \\
\hline \multicolumn{2}{|l|}{ Dialysis Dose Delivered } \\
\hline $\mathrm{Kt} / \mathrm{V}(\mathrm{std}, 87 \mathrm{pts})$ & $1.628 \pm 0.35$ \\
\hline Kt/V (Weekly, 5 pts) & $2.25 \pm 0.21$ \\
\hline Convective volume (L/session) & $24.80 \pm 3.05$ \\
\hline Residual Urinary Output $(\mathrm{n}>300 \mathrm{~mL} /$ day $)$ & $50(57.47 \%)$ \\
\hline \multicolumn{2}{|l|}{ Blood Pressure $(\mathrm{BP}) *(\mathrm{mmHg})$} \\
\hline Pre-dialysis Systolic BP & $141.71 \pm 22.66$ \\
\hline Pre-dialysis Diastolic BP & $68.43 \pm 15.26$ \\
\hline Post-dialysis Systolic BP & $134.74 \pm 20.5$ \\
\hline Post-dialysis Diastolic BP & $63.92 \pm 13.83$ \\
\hline \multicolumn{2}{|l|}{ Fluid status (bioimpedence measurement) } \\
\hline Fluid Overload (L) & $1.31 \pm 2.09$ \\
\hline Dry weight $(\mathrm{kg})$ & $75.75 \pm 18.02$ \\
\hline Ultrafiltation (mL/session) & $2090.3 \pm 928.77$ \\
\hline Ultrafiltation $(\mathrm{mL} / \mathrm{h} / \mathrm{kg})$ & $7.22 \pm 3.22$ \\
\hline \multicolumn{2}{|l|}{ Nutrition, Anemia \& Inflammation Markers } \\
\hline nPCR (g/kg/24 h) & $1.05 \pm 0.22$ \\
\hline Hemoglobin level (g/L) & $11.26 \pm 1.28$ \\
\hline Ferritin $(\mu \mathrm{g} / \mathrm{L})$ & $470.7 \pm 228.29$ \\
\hline Transferin Saturation coefficient (\%) & $26.84 \pm 12.49$ \\
\hline Albumin $(g / L)$ & $39.88 \pm 3.33$ \\
\hline C-reactive protein $(\mathrm{mg} / \mathrm{L})$ & $7.36 \pm 9.73$ \\
\hline
\end{tabular}

Table 3. Reported adverse events after vaccine.

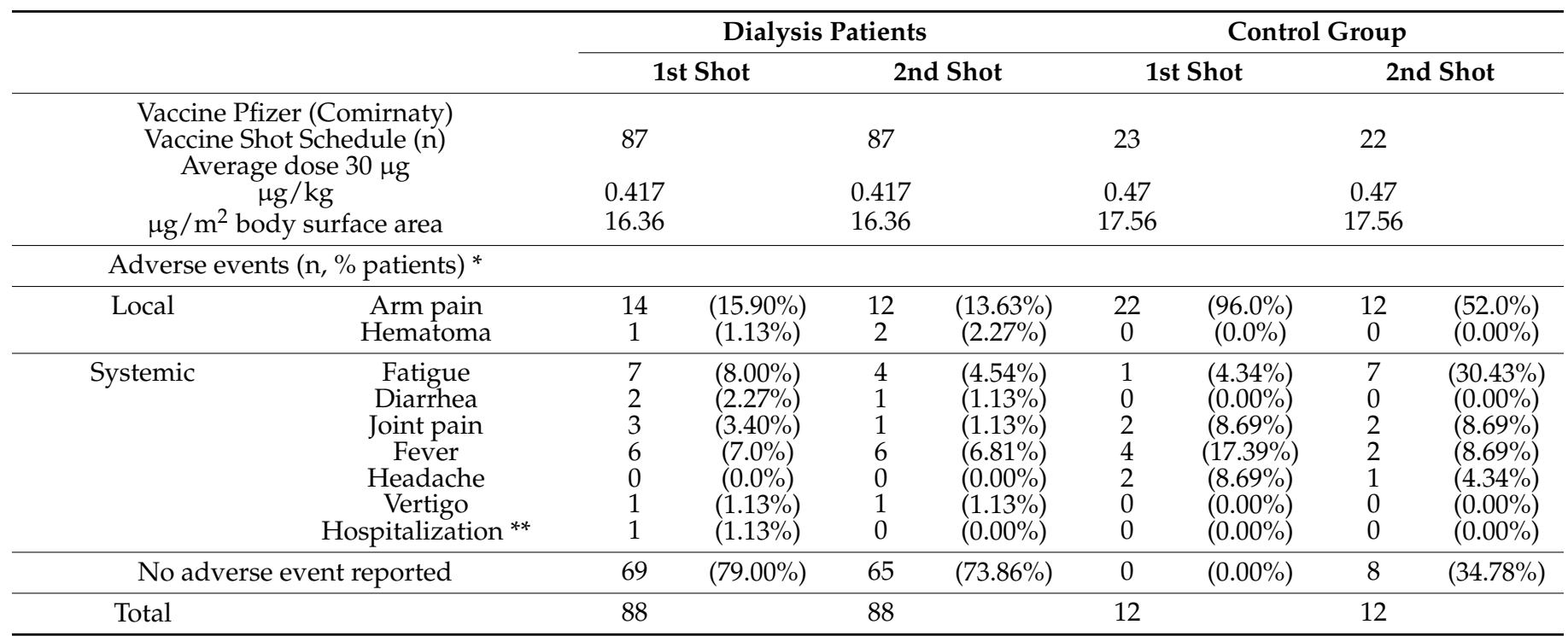

*: Several possible adverse events reported per patient/volunteer. ${ }^{* *}$ : Severe adverse event with Emergency Care Unit hospitalization. 


\subsection{Tolerability of the SARS-CoV-2 mRNA Vaccine}

Adverse effects of the anti-SARS-CoV-2 vaccine observed in HD patients and the control group are presented in Table 3. Hemodialysis patients displayed fewer symptoms than the control group. As shown, pain was noted in $16 \%$ versus $96 \%$ and in $13 \%$ versus $52 \%$ after the first and second vaccine shots, respectively. Fever reactions were noted in $7 \%$ versus $17 \%$ after the first vaccine shot. Two patients developed serious symptoms that included fever $\left(\geq 39^{\circ} \mathrm{C}\right)$, diarrhea and headache after the first vaccine shot. The first patient was admitted to an emergency unit and monitored for $24 \mathrm{~h}$. The fever persisted more than 3 days and laboratory tests identified acute inflammation with CRP $>30 \mathrm{mg} / \mathrm{L}$. The second patient developed flu-like symptoms that lasted $24 \mathrm{~h}$ and resolved with symptomatic treatment.

\subsection{Anti-S Antibody Titer Development after Vaccination in Nä̈ve HD Patients and Control Subjects}

As shown, mRNA vaccine administration induced a significant increase in antibody titers in both patients and healthy controls. One month after the first vaccine shot, $22(22.9 \%)$ patients and $4(17.39 \%)$ healthy subjects displayed positive nucleocapsid $(\mathrm{N})$ antibodies due to a previous contact with SARS-CoV-2 (Table 4). One month after the first vaccine shot, $64.3 \%$ of patients had a seroconversion, with anti-S antibody titers remaining between 0.4 and $50 \mathrm{U} / \mathrm{mL}, 11.5 \%$ had a positive response with anti-S antibody titers $\geq 50 \mathrm{U} / \mathrm{mL}$, and $24 \%$ of patients had a high positive response with anti-S antibody titers $>250 \mathrm{U} / \mathrm{mL}$. One month after the second vaccine shot, $96.5 \%$ had a boosted positive response, with anti-S antibody titers reaching $>250 \mathrm{U} / \mathrm{mL}$ (Figure $2 \mathrm{~A}-\mathrm{C}$ ). Two patients developed a symptomatic infection two weeks after the first vaccine shot, and consequently, the second shot was cancelled. In addition, an 81-year-old patient developed a severe symptomatic COVID-19 infection 2 weeks after the second shot and died in the intensive care unit. In this later case, no immune response was observed, as indicated by the lack of changes in anti-S antibody titers.

Table 4. Vaccine response.

\begin{tabular}{|c|c|c|c|c|c|c|c|c|c|}
\hline & \multicolumn{3}{|c|}{ Baseline } & \multicolumn{3}{|c|}{ 1st Shot } & \multicolumn{3}{|c|}{ 2nd Shot } \\
\hline & Patients & $\begin{array}{l}\text { Control } \\
\text { Group }\end{array}$ & & Patients & $\begin{array}{l}\text { Control } \\
\text { Group }\end{array}$ & & Patients & $\begin{array}{l}\text { Control } \\
\text { Group }\end{array}$ & \\
\hline \multicolumn{10}{|c|}{ Antibodies status before vaccination * } \\
\hline $\begin{array}{c}\text { IgG positive } \\
\text { (\% individuals) }\end{array}$ & $17.24 \%$ & $18 \%$ & & & & & & & \\
\hline $\begin{array}{l}\text { IgM positive } \\
\text { (\% individuals) }\end{array}$ & $10.34 \%$ & $18 \%$ & & & & & & & \\
\hline \multicolumn{10}{|c|}{ Antibodies response to vaccination $* *$} \\
\hline \multicolumn{10}{|l|}{ Anti-N (cutoff index) } \\
\hline$\%$ individuals $<1$ & & & & $77.01 \%$ & $82.61 \%$ & & $75.86 \%$ & $82.61 \%$ & \\
\hline$\%$ individuals $>1$ & & & & $22.99 \%$ & $17.39 \%$ & & $24.14 \%$ & $17.39 \%$ & \\
\hline \multicolumn{10}{|l|}{ Anti-S titers $(\mathrm{U} / \mathrm{mL})$} \\
\hline$\%$ individuals $<0.4$ & & & & $4.6 \%$ & $0 \%$ & & $2.3 \%$ & $0 \%$ & \\
\hline$\%$ individuals $0.4-50$ & & & & $59.77 \%$ & $34.78 \%$ & & $1.15 \%$ & $0 \%$ & \\
\hline$\%$ individuals $50-250$ & & & & $11.49 \%$ & $39.13 \%$ & & $2.3 \%$ & $4.35 \%$ & \\
\hline$\%$ individuals $>250$ & & & & $24.14 \%$ & $26.09 \%$ & & $94.25 \%$ & $95.65 \%$ & \\
\hline Blood cells parameters & \multicolumn{2}{|c|}{ Mean } & $95 \% \mathrm{CI}^{\S}$ & \multicolumn{2}{|c|}{ Mean } & $95 \% \mathrm{CI}^{\S}$ & \multicolumn{2}{|c|}{ Mean } & $95 \% \mathrm{CI}^{\S}$ \\
\hline Hemoglobin (g/dL) & 11.01 & 13.13 & $(1.49,2.75)$ & 11.26 & 13.47 & $(1.6,2.8)$ & 11.4 & 13.1 & $(1.2,2.2)$ \\
\hline Platelets $\left(\mathrm{n} / \mathrm{mm}^{3}\right)$ & 222,207 & 253,391 & $(-2305,64,674)$ & 228,288 & 251,087 & $(-12,349,57,948)$ & 242,044 & 266,870 & $(-36,296,85,947)$ \\
\hline $\begin{array}{l}\text { White Blood Cells } \\
\text { Count }\left(\mathrm{n} / \mathrm{mm}^{3}\right)\end{array}$ & 6289.2 & 7163.0 & $(-4.12,1751.8)$ & 6178.3 & 7468.7 & $(369,2212)$ & 6001 & 7129.6 & $(287,1971)$ \\
\hline Monocytes & 528.2 & 455.4 & $(10.66,134.96)$ & 527.1 & 453.5 & $(-10.8,158.0)$ & 525.7 & 494.4 & $(-41.2,103.9)$ \\
\hline Lymphocytes & 1409.4 & 2435.6 & $(673,1379)$ & 1462.1 & 2500.7 & $(506,1571)$ & 1351.4 & 2968.9 & $(1037,2199)$ \\
\hline $\begin{array}{l}\% \text { individuals }< \\
1000 / \mathrm{mm}^{3}\end{array}$ & $17.24 \%$ & $0.00 \%$ & & $26.44 \%$ & $8.7 \%$ & & $35.63 \%$ & $0.00 \%$ & \\
\hline
\end{tabular}

*: IgG and IgM: Abbott; **: Anti-N and Anti-S antibodies: Elecsys ${ }^{\circledR}$ (Roche); $§$ : 95\% CI for the difference of the two means. 

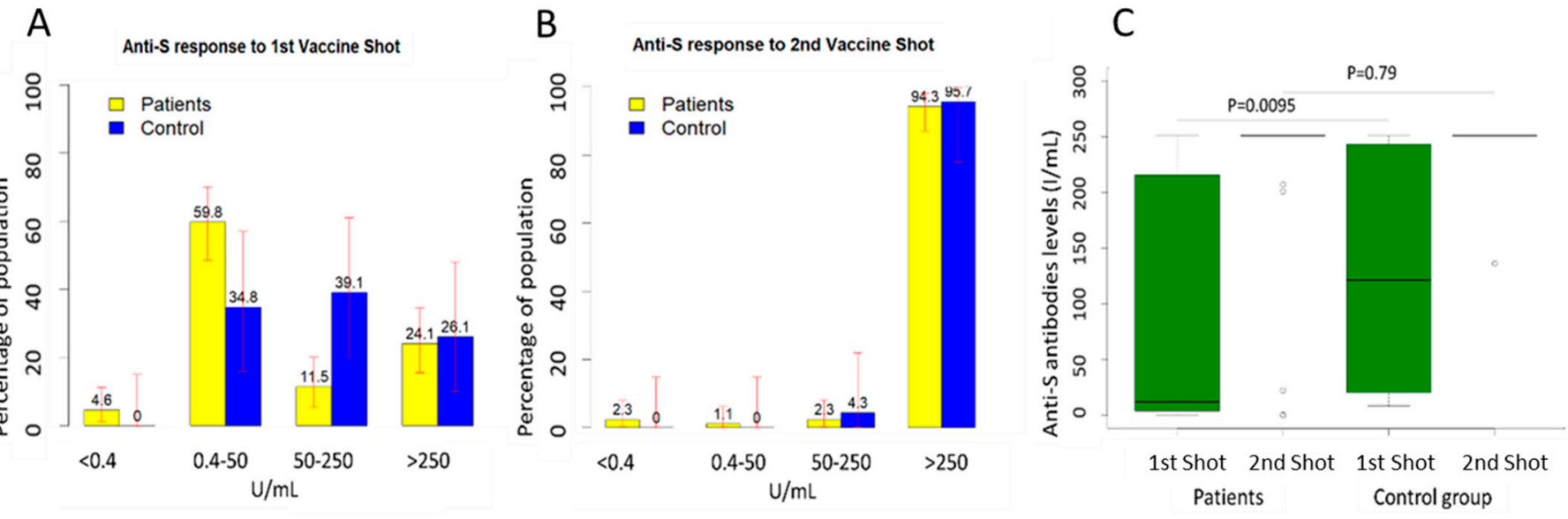

Figure 2. Comparison between patients and control group for Anti-S antibodies response after the 1st vaccine shot. (A) Comparison between patients and control group for Anti-S antibodies response after the 1st vaccine shot. (B) Comparison between patients and control group for Anti-S antibodies response after the 2 nd vaccine shot. (C) Comparison between naïve groups: patients vs controls for Anti-S antibodies response after 1st and 2nd vaccine shots.

\subsection{Anti-S Antibody Titer Development in HD Patients Previously Exposed to SARS-CoV-2}

After the first vaccine shot, $95 \%$ of HD patients who had contracted COVID-19 prior to their vaccination developed high levels of anti-S antibodies $(>250 \mathrm{U} / \mathrm{mL})$ comparable to the control group. The second shot in this subgroup did not further increase the titer of anti-S antibodies (Figure 3). Figure 2 shows a comparison between patients and the control group of vaccine response after first and second vaccine shots. Figure 3 shows a comparison of vaccine response after the first and second vaccine shots according to each patient's SARS-CoV-2 virus exposure status.
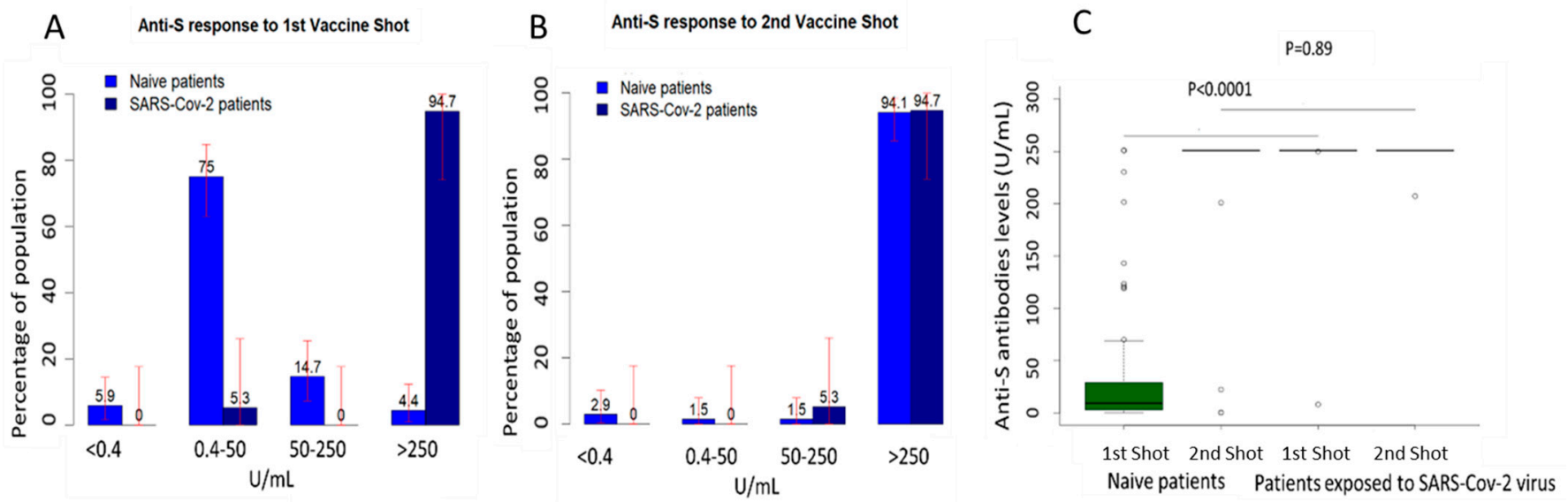

Figure 3. Comparison of vaccine response after first and second vaccine shots according to patients SARS-CoV-2 virus exposure status. (A) Comparison of Anti-S antibodies response after 1st vaccine shot between naïve and SARS-CoV-2 patients. (B) Comparison of Anti-S antibodies response after 2 nd vaccine shot between naïve and SARS-CoV-2 patients. (C) Anti-S antibodies response after 1st and 2 nd vaccine shots according to the SARS-CoV-2 virus exposure status.

\subsection{Relative Fluid Overload (FO) and Anti-S Antibodies in Nä̈ve Dialysis Patients}

Relative overload evaluated from multifrequency bioimpedance $\left(\mathrm{BCM}^{\circledR}\right)$ is defined in our clinical practice as $>15 \%$ for men and $>13 \%$ for women when compared to a reference normovolemic population [11]. In Table 5, anti-S antibody titers are scattered according to patient fluid status and clustered into three categories (normovolemic $<1.5 \mathrm{~L}$, mild fluid overload (1.5-3 L) and severe fluid overload $>3 \mathrm{~L}$ ). The median antibody titers were 
much higher in normovolemic than in FO patients after the first vaccine shot. As is shown, the percentage of patients with protective anti-S antibody titers decreased gradually with increasing FO. Anti-S antibody titers tended to be inversely correlated with relative fluid overload in HD patients but did not reach statistical significance. This is likely due to the relatively low prevalence of fluid overload.

Table 5. Hemodialysis conditions and vaccine response according to patient's fluid overload status.

\begin{tabular}{|c|c|c|c|}
\hline & $\begin{array}{l}\text { Normovolemic } \\
\begin{array}{c}(<+1.5 \mathrm{~L}) \\
\mathrm{n}=49\end{array}\end{array}$ & $\begin{array}{l}\text { Mild Fluid Overload } \\
\begin{array}{c}(1.5-3 \mathrm{~L}) \\
\mathrm{n}=25\end{array}\end{array}$ & $\begin{array}{l}\text { Severe Fluid Overload } \\
\qquad \begin{array}{c}(>3 \mathrm{~L}) \\
\mathrm{n}=13\end{array}\end{array}$ \\
\hline Fluid Overload (L) & $\begin{array}{c}\text { Median (25th-75th percentile) } \\
0.49(-0.34-0.95)\end{array}$ & $2(1.89-2.44)$ & $4.33(3.64-4.99)$ \\
\hline $\begin{array}{l}\text { Number of patients with urine output } \\
>300 \mathrm{~mL} / \text { day }\end{array}$ & $\begin{array}{c}\text { Number of patients (\%) } \\
25(51 \%)\end{array}$ & $16(64 \%)$ & $11(85 \%)$ \\
\hline $\begin{array}{c}\text { Dialysis conditions } \\
\text { HD } \\
\text { HDF } \\
\text { Home Daily Hemodialysis }\end{array}$ & $\begin{array}{l}\text { Number of patients (\% patients) } \\
28(57 \%) \\
17(35 \%) \\
4(8 \%)\end{array}$ & $\begin{array}{c}11(44 \%) \\
13(52 \%) \\
1(4 \%)\end{array}$ & $\begin{array}{l}6(46 \%) \\
7(54 \%) \\
0(0 \%)\end{array}$ \\
\hline $\begin{array}{c}\text { Dialysis performance } \\
\text { Ultrafiltration Rate }(\mathrm{mL}) \\
\mathrm{Kt} / \mathrm{V}\end{array}$ & $\begin{array}{c}\text { Median (25th-75th percentile) } \\
1760(1200-2377) \\
1.58(1.3-1.85)\end{array}$ & $\begin{array}{c}2000(1617-2490) \\
1.59(1.46-1.82)\end{array}$ & $\begin{array}{l}1800(1485-2804) \\
1.66(1.39-1.8)\end{array}$ \\
\hline $\begin{array}{l}\text { SARS-CoV-2 vaccine response } \\
\text { Anti-S after 1st Shot }(\mathrm{U} / \mathrm{mL}) \\
\text { Anti-S after } 2 \text { nd Shot }(\mathrm{U} / \mathrm{mL})\end{array}$ & $\begin{array}{c}\text { Median (25th-75th percentile) } \\
23.9(4.99->250) \\
>250(>250->250)\end{array}$ & $\begin{array}{l}11.54(2.95-121.4) \\
>250(>250->250)\end{array}$ & $\begin{array}{l}9.43(1.59-22.42) \\
>250(207.3->250)\end{array}$ \\
\hline $\begin{array}{l}\text { Levels of vaccine response * } \\
\text { After 1st Shot: }<50 \mathrm{U} / \mathrm{mL} \\
<250 \mathrm{U} / \mathrm{mL} \\
\text { After 2nd Shot: }<50 \mathrm{U} / \mathrm{mL} \\
<250 \mathrm{U} / \mathrm{mL}\end{array}$ & $\begin{array}{l}\text { Number of patients (\% patients) } \\
28(57 \%) \\
35(71 \%) \\
1(2 \%) \\
2(4 \%)\end{array}$ & $\begin{array}{c}17(68 \%) \\
19(76 \%) \\
1(4 \%) \\
1(4 \%)\end{array}$ & $\begin{array}{c}11(85 \%) \\
13(100 \%) \\
1(8 \%) \\
2(15 \%)\end{array}$ \\
\hline $\begin{array}{c}\text { Hepatitis B vaccine response } \\
\text { Levels of vaccine response } * * \\
\text { Anti HBS }<10 \mathrm{IU} / \mathrm{L} \\
\text { Anti } \mathrm{HBS}<100 \mathrm{IU} / \mathrm{L}\end{array}$ & Number of patients (\% patients) & $\begin{array}{c}3(12 \%) \\
13(52 \%)\end{array}$ & $\begin{array}{l}1(8 \%) \\
6(46 \%)\end{array}$ \\
\hline $\begin{array}{c}\text { Laboratory data } \\
\text { Albumin } \\
\text { C-Reactive Protein (CRP) }\end{array}$ & $\begin{array}{c}\text { Median (25th-75th percentile) } \\
41(37-42) \\
6(2.8-10.7)\end{array}$ & $\begin{array}{c}38(36-40) \\
5.6(3.5-9.9)\end{array}$ & $\begin{array}{l}39(38-41) \\
10(6-10.3)\end{array}$ \\
\hline
\end{tabular}

*: $<50 \mathrm{U} / \mathrm{mL}$ : no seroprotection according to ELISA assay (Roche), $<250 \mathrm{U} / \mathrm{mL}$ : low seroprotection according to WHO recommendations. ${ }^{* *}$ : <10 IU/L: No seroconversion, <100 IU/L: no seroprotection.

\section{Discussion}

Vaccination is considered the most effective health measure to tackle the ongoing global COVID-19 pandemic. Chronic Kidney Dialysis (CKD) patients are more susceptible to infections due to their immunocompromised profile, as reflected by a defective vaccine response to BCG, influenza, and hepatitis viruses [12]. In addition, patients on hemodialysis (HD) are particularly susceptible to develop more severe forms of COVID-19 with a higher risk of death $[13,14]$. In this study, we reported several original findings: First, we observed both safety and excellent tolerability of the Comirnaty ${ }^{\mathrm{TM}}$, Pfizer/BioNtech SARS-CoV-2 mRNA vaccine in the vulnerable HD population. Second, our study highlights the efficacy and the immunogenic profile of the SARS-CoV-2 mRNA vaccine which elicits in most patients $(>98 \%)$ a significant humoral immune response against the SARS-CoV-2 spike protein after complete vaccination, with a higher seroconversion rate than the hepatitis $B$ vaccine. Third, here we reveal two major factors of variability affecting the vaccine response by showing that pre-exposure of HD patients to SARS-CoV-2 boosted anti-spike antibody 
levels, while on the contrary fluid overload (FO) appears as a new inhibitory host factor that may hamper the anti-S antibody response in HD patients.

Vaccines are intended to induce the host immune response, and protection may induce side effects related to their potent systemic inflammatory action [15]. In comparison to the healthy controls, the HD patients displayed less pain, and fever, which are major hallmarks of local and systemic inflammation. In addition, HD patients had defects within their innate immune cells, which are known as key cellular mediators in the systemic inflammatory reaction [13] that may explain less side effects observed in vaccinated HD patients.

Altogether, our findings highlight that the mRNA SARS-CoV-2 vaccine elicits both safety and great tolerability among HD patients.

Naïve HD patients developed a significant adaptive immune response against the SARS-CoV-2 spike protein following complete vaccination, consisting of a strong antibody response after the second shot comparable to the control group. Indeed, we report an effective antibody response $(>50 \mathrm{U} / \mathrm{mL})$ in nearly $98 \%$ of these patients.

In line with the reduced vaccine response described in advanced CKD patients, H1N1 vaccination during the 2009 pandemic led to seroconversion in only $57 \%$ to $64.2 \%$ of $\mathrm{HD}$ patients, where the well-known subunit vaccine Pendemrix was used [16]. Here, we report that on the contrary the mRNA vaccine triggered a robust antibody immune response that consisted of a higher seroconversion rate than the hepatitis $B$ vaccine. Two dialysis patients who were not vaccinated during the vaccination campaign developed a symptomatic form of COVID-19 infection; three other vaccinated patients developed an asymptomatic form of COVID-19 disease.

Altogether, these findings suggest that the mRNA vaccine against SARS-CoV-2 is immunogenic and can elicit protective immunity in HD patients. Further studies with larger cohorts of HD patients are needed to evaluate potential vaccine schemes to achieve better protection to prevent severe COVID-19 disease forms.

Interestingly, one patient, aged 46 years, did not develop an anti-S antibody response. The lack of an immune response in that case was due to a cellular immune deficiency, with a CD4 T cell count less than $200 / \mathrm{mm}^{3}$, suggesting the importance of T cell Help or assistance for an optimal antibody response [17].

From a mechanistic point of view, the ability of the mRNA vaccine to elicit a potential protective $\mathrm{B} / \mathrm{T}$ cell immunity may be explained by its higher immunogenic profile due to two innovative properties: first, its self-adjuvanticity action; second, its biochemical formulation and engineering relying on lipidic nanoparticles (LNPs) inclusion that prevents degradation and facilitates their efficient capture by antigen-presenting cells (APC) such as dendritic cells (DCs) $[18,19]$. In addition, mRNA LNPs can induce DC activation and type 1 interferon expression in DCs, demonstrating that they can promote a potent anti-viral response $[20,21]$.

Hence, the mRNA vaccine shows self-adjuvanticity that induces both humoral and cellular immune response against the encoded antigenic protein such as spike protein [22]. Accordingly, DCs are key players for vaccine-mediated protective responses via their unique ability to induce generation of long-lived memory antigen-specific $\mathrm{T}$ and $\mathrm{B}$ cells by presenting antigens to both T cells [23] and B cells [24]. The central role of DCs in vaccine-induced immune response was supported in a recent study of El-Barbry et al. [24] showing that DCs can capture and release antigens that are sufficient to promote early B cell activation both in vivo and in vitro, that can be associated with a potential plasma cell differentiation [24].

In this study, we reveal that two major factors of variability may affect and modulate the vaccine response in HD patients both positively and negatively. First, we show that pre-exposure of HD patients to SARS-CoV-2 boosted anti-spike antibody levels. Indeed, we observed that HD patients who previously contracted COVID-19 were able to generate comparable levels of anti-spike antibodies to the control group just after the first shot. These findings indicate that HD patients previously infected with SARS-CoV-2 develop an efficient immune memory response that can be re-activated upon vaccination. 
In contrast, our findings seem to indicate that fluid overload (FO) appears as a new inhibitory host factor that may hamper the anti-S antibody response in HD patients.

It is well known that HD patients commonly suffer from various complications, such as a hyper uremic state [25], altered microbiota [26], compromised nutritional statuses [27] and fluid overload [28] that increase their risk of infection. It is also known that aging is inversely correlated with the immune response upon vaccination against SARS-CoV-2 using the Pfizer/BioNTech mRNA vaccine [29]. Loss of efficacy in vaccination across CKD stages relies on multiple disorders that affect both innate and adaptive immunity $[28,30]$. The accumulation of uremic toxins in HD patients tends to alter the functions of dendritic cells (DCs) and innate cells, such as monocytes and neutrophils [28,31]. Dendritic cells (DCs) are migratory phagocytes that play a key role in immunity upon respiratory lung infection [31].

A common feature in advanced CKD and HD patients is fluid overload (FO), which is associated with multiple biological and pathophysiological consequences. It is an independent biomarker and a risk factor involved in the development of cardiovascular morbidities such as congestive heart failure and left ventricular hypertrophy [32]. It was previously shown in the literature that FO-associated cardiovascular impairment includes the secretion of brain natriuretic peptide (BNP), decreasing in the total monocytes, B cells, and natural killer cells and then impairing natural killer cell cytotoxicity [33]. Additionally, FO is associated with immune deficiency and a higher burden of inflammation compared to normal individuals [28]. This cardiorenal crosstalk impairment triggers the release of proinflammatory cytokines, such as tumor necrosis factor alpha (TNF- $\alpha$ ) and interleukins (IL)-1 and IL-6, which, in turn, contribute to aggravate salt and water retention [34]. Accordingly, HD patients may exhibit a vicious cycle of inflammatory-fluid overload that negatively affects their innate and adaptive immune responses [35].

The findings of our study agree with these results, suggesting that FO may lead to cardiac decompensation but may also hamper the immune response to microbiologic agents in HD patients. Indeed, previous reports have shown that immune dysfunction increases the risk of pulmonary infection two-fold [36], magnifies the severity of symptoms and increases mortality risk in CKD patients [37]. Our study reports that anti-S antibody titers are inversely correlated with fluid overload in a negative-like volume effect.

As suggested by our findings, fluid overload, promoting a chronic low-grade inflammatory state in HD patients, may represent a novel host negative checkpoint that impedes the humoral immune response to vaccination in our HD patient cohort. FO should be considered as a contributing factor to the acquired immunodeficiency of HD patients. Larger comparative studies are needed to confirm or not our preliminary results on the negative impact of FO in altering patients' immune response.

Dialysis modality may also influence vaccine response. In a recent study, it was shown that dialysis patients might exhibit similar percentages of seroconversion against the trivalent inactivated influenza vaccine, not exceeding the $50 \%$ of seroconversion for the H1N1 vaccine. Interestingly, the authors showed that seroconversion was maintained longer, and the lymphocyte proliferation rate was better in patients undergoing hemodiafiltration compared to HD patients. These findings suggested that hemodiafiltration more efficiently cleared middle and large molecular weight uremic toxins compared to high-flux hemodialysis, which may contribute to improve immune response [9].

However, our study has some limitations. The cohort of HD patients is limited, and HD patients suffer from hypertension and other comorbidities whereas the control groups are healthy. The age of HD patients is higher than the control groups. Anti-S antibody titers tended to be inversely correlated with relative FO in HD patients but did not reach statistical significance. This is likely due to the relatively low prevalence of fluid overload patients in our cohort.

Our study provides very encouraging results by showing that the new generation mRNA vaccine approach is safe, efficient and may be used in HD patients. It highlights the importance of factors that should be addressed to ensure successful vaccination in these 
patients, such as fluid overload. It is a new marker that should be monitored to improve the vaccination of HD patients because water overload impedes the immune system. Further and larger studies are needed to validate our findings on the trend of negative impact on the vaccine immune response of HD patients. Additionally, cell profiling and better phenotyping and functional characterization of T cell subtypes in vaccinated HD patients are needed.

Author Contributions: Conception, design, analysis, and interpretation: H.H., M.E., L.C., F.O., B.C. Data collection and writing the article: H.H., M.E., B.C., D.A., F.O., L.C. Critical revision of the article: H.H., M.E., D.A., L.C., S.S., S.B., J.A., C.C., F.O., B.C. Overall responsibility: H.H. All authors have read and agreed to the published version of the manuscript.

Funding: This research received no external funding.

Institutional Review Board Statement: The study was conducted according to the guidelines of the Declaration of Helsinki, and approved by the Institutional Review Board of Fresenius Medical Care and NephroCare France.

Informed Consent Statement: Informed consent was obtained from all subjects involved in the study.

Conflicts of Interest: The authors declare no conflict of interest.

\section{References}

1. Flythe, J.E.; Assimon, M.M.; Tugman, M.J.; Chang, E.H.; Gupta, S.; Shah, J.; Sosa, M.A.; Renaghan, A.D.; Melamed, M.L.; Perry Wilson, F.; et al. Characteristics and Outcomes of Individuals with Pre-existing Kidney Disease and COVID-19 Admitted to Intensive Care Units in the United States. Am. J. Kidney Dis. 2021, 77, 190-203. [CrossRef] [PubMed]

2. De Meester, J.; de Bacquer, D.; Naesens, M.; Meijers, B.; Couttenye, M.M.; de Vriese, A.S.; NBVN Kidney Registry Group. Incidence, characteristics, and outcome of COVID-19 in adults on kidney replacement therapy: A regionwide registry study. J. Am. Soc. Nephrol. 2021, 32, 385-396. [CrossRef]

3. Hsu, C.M.; Weiner, D.E.; Aweh, G.; Miskulin, D.C.; Manley, H.J.; Stewart, C.; Ladik, V.; Hosford, J.; Lacson, E.C.; Johnson, D.S.; et al. COVID-19 Infection Among US Dialysis Patients: Risk Factors and Outcomes from a National Dialysis Provider. Am. J. Kidney Dis. 2021, 77, 748-756. [CrossRef]

4. $\quad$ Ng, J.H.; Hirsch, J.S.; Wanchoo, R.; Sachdeva, M.; Sakhiya, V.; Hong, S.; Jhaveri, K.D.; Fishbane, S.; Northwell COVID-19 Research Consortium and the Northwell Nephrology COVID-19 Research Consortium. Outcomes of patients with end-stage kidney disease hospitalized with COVID-19. Kidney Int. 2020, 98, 1530-1539. [CrossRef] [PubMed]

5. Zitt, E.; Davidovic, T.; Schimpf, J. The Safety and Immunogenicity of the mRNA-BNT162b2 SARS-CoV-2 Vaccine in Hemodialysis Patients. Front. Immunol. 2021, 12, 704773. [CrossRef] [PubMed]

6. Boyarsky, B.J.; Werbel, W.A.; Avery, R.K.; Tobian, A.A.R.; Massie, A.B.; Segev, D.L.; Garonzik-Wang, J.M. Immunogenicity of a Single Dose of SARS-CoV-2 Messenger RNA Vaccine in Solid Organ Transplant Recipients. JAMA 2021, 325, 1784-1786. [CrossRef]

7. Polack, F.P.; Thomas, S.J.; Kitchin, N.; Absalon, J.; Gurtman, A.; Lockhart, S.; Perez, J.L.; Pérez Marc, G.; Moreira, E.D.; Zerbini, C.; et al. Safety and Efficacy of the BNT162b2 mRNA COVID-19 Vaccine. N. Engl. J. Med. 2020, 383, $2603-2615$. [CrossRef] [PubMed]

8. Weiss, S.; Bhat, P.; Del Pilar Fernandez, M.; Bhat, J.G.; Coritsidis, G.N. COVID-19 Infection in ESKD: Findings from a Prospective Disease Surveillance Program at Dialysis Facilities in New York City and Long Island. J. Am. Soc. Nephrol. 2020, 31, $2517-2521$. [CrossRef]

9. Nongnuch, A.; Ngampongpan, W.; Srichatrapimuk, S.; Wongsa, A.; Thongpraphai, S.; Boonarkart, C.; Sanmeema, N.; Chittaganpitch, M.; Auewarakul, P.; Tassaneetrithep, B.; et al. Immune response to influenza vaccination in ESRD patients undergoing hemodialysis vs. hemodiafiltration. PLOS ONE 2020, 15, e0227719.

10. Clarke, C.; Prendecki, M.; Dhutia, A.; Ali, M.A.; Sajjad, H.; Shivakumar, O.; Lightstone, L.; Kelleher, P.; Pickering, M.C.; Thomas, D.; et al. High Prevalence of Asymptomatic COVID-19 Infection in Hemodialysis Patients Detected Using Serologic Screening. J. Am. Soc. Nephrol. 2020, 31, 1969-1975. [CrossRef]

11. Zoccali, C.; Moissl, U.; Chazot, C.; Mallamaci, F.; Tripepi, G.; Arkossy, O.; Wabel, P.; Stuard, S. Chronic Fluid Overload and Mortality in ESRD. J. Am. Soc. Nephrol. 2017, 28, 2491-2497. [CrossRef] [PubMed]

12. Haddiya, I. Current Knowledge of Vaccinations in Chronic Kidney Disease Patients. Int. J. Nephrol. Renovasc. Dis. 2020, 13, 179-185. [CrossRef] [PubMed]

13. ERA-EDTA Council, ERACODA Working Group. Chronic kidney disease is a key risk factor for severe COVID-19: A call to action by the ERA-EDTA. Nephrol. Dial. Transplant. 2021, 36, 87-94. [CrossRef] [PubMed]

14. Eleftheriadis, T.; Antoniadi, G.; Liakopoulos, V.; Kartsios, C.; Stefanidis, I. Basic science and dialysis: Disturbances of acquired immunity in hemodialysis patients. Semin. Dial. 2007, 20, 440-451. [CrossRef] 
15. Hervé, C.; Laupèze, B.; del Giudice, G.; Didierlaurent, A.M.; da Silva, F.T. The how's and what's of vaccine reactogenicity. NPJ Vaccines 2019, 4, 39. [CrossRef]

16. Pollard, A.J.; Bijker, E.M. A guide to vaccinology: From basic principles to new developments. Nat. Rev. Immunol. 2021, 21, 83-100. [CrossRef]

17. Broeders, N.E.; Hombrouck, A.; Lemy, A.; Wissing, K.M.; Racapé, J.; Gastaldello, K.; Massart, A.; van Gucht, S.; Weichselbaum, L.; de Mul, A.; et al. Influenza A/H1N1 vaccine in patients treated by kidney transplant or dialysis: A cohort study. Clin. J. Am. Soc. Nephrol. 2011, 6, 2573-2578. [CrossRef]

18. Batty, C.; Bachelder, E.M.; Ainslie, K.M. Historitical Perspective of Clinical Nano and Microparticle Formulation for Delivery of Therapeutics. Forum Ser. Bottlenecks Breakthr. Mol. Med. 2021, 27, 516-519.

19. Wang, Y.; Zhang, Z.; Luo, J.; Han, X.; Wei, Y.; Wei, X. mRNA vaccine: A potential therapeutic strategy. Mol. Cancer 2021, 20, 33. [CrossRef]

20. Liang, F.; Lindgren, G.; Lin, A.; Thompson, E.A.; Ols, S.; Röhss, J.; John, S.; Hassett, K.; Yuzhakov, O.; Bahl, K.; et al. Efficient targeting and activation of antigen-presenting cells in vivo after modified mRNA vaccine administration in rhesus macaques. Mol. Ther. 2017, 25, 2635-2647. [CrossRef]

21. Pardi, N.; Hogan, M.J.; Porter, F.W.; Weissman, D. mRNA vaccines-A new era in vaccinology. Nat. Rev. Drug Discov. 2018, 17, 261. [CrossRef]

22. Pepini, T.; Pulichino, A.; Carsillo, T.; Carlson, A.L.; Sari-Sarraf, F.; Ramsauer, K.; Debasitis, J.C.; Maruggi, G.; Otten, G.R.; Geall, A.J.; et al. Induction of an IFN-Mediated Antiviral Response by a Self-Amplifying RNA Vaccine: Implications for Vaccine Design. J. Immunol. 2017, 198, 4012-4024. [CrossRef] [PubMed]

23. Guermonprez, P.; Saveanu, L.; Kleijmeer, M.; Davoust, J.; Endert Van, P.; Amigorena, S. ER-phagosome fusion defines an MHC class I cross-presentation compartment in dendritic cells. Nature 2003, 425, 397-402. [CrossRef] [PubMed]

24. El-Barbry, H.; Capitao, M.; Barrin, S.; Amziani, S.; Paul, P.P.; Borreill, S.; Guilbert, T.; Donnadieu, E.; Niedergang, F.; Ouaaz, F. Extracellular Release of Antigen by Dendritic Cell Regurgitation Promotes B Cell Activation through NF-kB/cRel. J. Immunol. 2020, 205, 608-618. [CrossRef] [PubMed]

25. Yoshida, H.; Inaguma, D.; Koshi-Ito, E.; Ogata, S.; Kitagawa, A.; Takahashi, K.; Koide, S.; Hayashi, H.; Hasegawa, M.; Yuzawa, Y.; et al. Extreme hyperuricemia is a risk factor for infection-related deaths in incident dialysis patients: A multicenter prospective cohort study. Ren. Fail. 2020, 42, 646-655. [CrossRef]

26. Durand, P.Y.; Nicco, C.; Serteyn, D.; Attaf, D.; Edeas, M. Microbiota Quality and Mitochondrial Activity Link with Occurrence of Muscle Cramps in Hemodialysis Patients using Citrate Dialysate: A Pilot Study. Blood Purif. 2018, 46, 301-308. [CrossRef]

27. Alvarenga, L.A.; Andrade, B.D.; Moreira, M.A.; Nascimento, R.P.; Macedo, I.D.; Aguiar, A.S. Nutritional profile of hemodialysis patients concerning treatment time. J. Bras. Nefrol. 2017, 39, 283-286. [CrossRef]

28. Ulrich, C.; Wilke, A.; Schleicher, N.; Girndt, M.; Fiedler, R. Hypervolemia-induced Disturbances Do Not involve IL-1 ss but IL-6 and IL-10 activation in hemodialysis patients. Toxins 2020, 12, 159. [CrossRef]

29. Grupper, A.; Sharon, N.; Finn, T.; Cohen, R.; Israel, M.; Agbaria, A.; Rechavi, Y.; Schwartz, I.F.; Schwartz, D.; Lellouch, Y.; et al. Humoral Response to the Pfizer BNT162b2 Vaccine in Patients Undergoing Maintenance Hemodialysis. Clin. J. Am. Soc. Nephrol. 2021, 16, 1037-1042. [CrossRef]

30. Hsu, H.-W.; Lang, C.-L.; Wang, M.-H.; Chiang, C.-K.; Lu, K.-C. A review of Chronic Kidney Disease and the Immune System: A special Form of Immunosenescence. J. Gerontol. Geriat. Res. 2014, 3, 1000144.

31. Cook, P.C.; MacDonald, A.S. Dendritic cells in Lung immunopathology. Semin. Immunopathol. 2016, 38, 449-460. [CrossRef] [PubMed]

32. Tsai, Y.-C.; Chiu, Y.-W.; Tsai, J.-C.; Kuo, H.-T.; Hung, C.-C.; Hwang, S.-J.; Chen, T.-H.; Kuo, M.-C.; Chen, H.-C. Association of fluid overload with cardiovascular morbidity and all-cause mortality in stages 4 and 5 CKD. Clin. J. Am. Soc. Nephrol. 2015, 10, 39-46. [CrossRef] [PubMed]

33. Shaw, S.M.; Fildes, J.E.; Puchałka, C.M.; Basith, M.; Yonan, N.; Williams, S.G. BNP directly regulates the innate immune system of cardiac transplant recipients in vitro. Transpl. Immunol. 2009, 20, 199-202. [CrossRef] [PubMed]

34. Azzam, Z.S.; Kinaneh, S.; Fadel Bahouth, F.; Ismael-Badarneh, R.; Khoury, E.; Abassi, Z. Involvement of Cytokines in the Pathogenesis of Salt and Water Imbalance in Congestive Heart Failure. Front. Immunol. 2017, 8, 716. [CrossRef]

35. Peesapati, V.S.R.; Sadik, M.; Verma, S.; Attallah, M.A.; Khan, S. Panoramic Dominance of the Immune System in Cardiorenal Syndrome Type, I. Cureus 2020, 12, e9869. [CrossRef]

36. Cohen-Hagai, K.; Rozenberg, I.; Korzets, Z.; Zitman-Gal, T.; Einbinder, Y.; Benchetrit, S. Upper Respiratory Tract Infection among Dialysis Patients. Isr. Med. Assoc. J. 2016, 18, 557-560.

37. Su, G.; Iwagami, M.; Qin, X.; McDonald, H.; Liu, X.; Carrero, J.J.; Lundborg, C.S.; Nitsch, D. Kidney disease and mortality in patients with respiratory tract infections: A systematic review and meta-analysis. Clin. Kidney J. 2021, 14, 602-611. [CrossRef] 